\title{
SLIP-DEPENDENT FRICTION IN DYNAMIC ELECTROVISCOELASTICITY
}

\author{
Mostafa Kabbaj and El-Hassan Essoufi \\ University Moulay Ismail, Morocco and University Hassan Premier, Morocco
}

\begin{abstract}
Existence and uniqueness of a weak solution for dynamical frictional contact between an electro-viscoelastic body and a rigid electrically non-conductive obstacle is established. The contact is modelled with a simplified version of Coulomb's law of dry friction in which the coefficient of friction depends on the slip. The proof is based on the regularization method, Faedo-Galerkin method, compactness and lower semicontinuity arguments.
\end{abstract}

\section{INTRODUCTION}

Piezoelectric materials are advanced materials and have many applications due to their electromechanical coupling effect. It is an example of phenomena where coupling two physical fields of different natures (namely mechanical and electrical fields) is the key factor to be taken into account in a variety of applications. The piezoelectricity can be described as follows: when mechanical pressure is applied to a certain classes of crystalline materials, the crystalline structure produces a voltage proportional to the pressure. Conversely, when an electric field is applied, the structure changes his shape producing dimensional modifications in the material. Actually, there is a big interest into the study of piezoelectric materials, this type of materials being used in radioelectronics, electroacoustics and measuring equipments. In the same time, due to the fact that the parts of the equipments are in contact, the interest for the contact problems is increasing. However, there are very few mathematical results concerning contact problems involving piezoelectric

2010 Mathematics Subject Classification. 37L65, 49J40, 74A55, 74D05, 74H20, 74H25.

Key words and phrases. Dynamic electroviscoelasticity, second-order hyperbolic variational inequality, regularization method, Faedo-Galerkin method, compactness method, existence, uniqueness. 
materials and therefore there is a need to extend the results on the models for contact with deformable bodies to models for piezoelectric contact. The literature concerning this topic is very rich, see from example $[1,5,9-11,14-16]$ for modelling in piezoelectricity or electro viscoelasticity. Some theoretical results for contact models taking into account the interaction between the electric and mechanic fields has been obtained in $[2,8,12,13]$. Recently, some dynamic contact problems for piezoelectric body were considered in $[4,7]$.

In this paper we study a dynamical frictional contact between a electroviscoelastic body which is acted upon by volume force and surface tractions, and a rigid electrically non-conductive foundation. So, we assume that the process is dynamic and use a simplified version of Coulomb's law in which the normal stress is prescribed and the coefficient of friction depends on the slip. We present weak formulation of the model which consists in a system coupling a variational inequality for the displacement field and a time-dependent variational equation for the electric field and show the existence and uniqueness of a weak solution to model. Such result extends the result obtained in [7] in which the authors trait the case where the frictional coefficient doesn't depend on the slip.

The rest of the paper is structured as follows. The model of the contact dynamic process of the linear electro viscoelastic body is presented in Section 2. In Section 3 we list the assumptions on the problem data, derive the variational formulation of the problem and state our main result, Theorem 3.1. The proof of the Theorem 3.1 is presented in Section 4. It is based on a regularization method, Galerkin method, compactness and lower semicontinuity arguments.

\section{Problem setting}

We consider a linear piezoelectric body that initially occupies a bounded domain $\Omega$ in $\mathbb{R}^{d}(d=2,3)$ with a smooth boundary $\Gamma$. Let $\boldsymbol{\nu}=\left(\nu_{i}\right)$ denote the unit outer normal on $\Gamma$ and $[0, T]$ be time interval of interest, where $T>0$. The indices $i, j, k, l$ run between 1 and $d$. The summation convention over repeated indices is adopted and the index that follows a comma indicates a partial derivative with respect to the corresponding component of the independent variable.

Everywhere below we use $\mathbb{S}^{d}$ to denote the space of second order symmetric tensors on $\mathbb{R}^{d}$ while "." and $|\cdot|$ will represent the inner product and the Euclidean norm on $\mathbb{R}^{d}$ and $\mathbb{S}^{d}$, that is: $\forall \boldsymbol{u}, \boldsymbol{v} \in \mathbb{R}^{d}, \quad \forall \boldsymbol{\sigma}, \boldsymbol{\tau} \in \mathbb{S}^{d}$

$\boldsymbol{u} \cdot \boldsymbol{v}=u_{i} v_{i}, \quad|\boldsymbol{v}|=(\boldsymbol{v} \cdot \boldsymbol{v})^{1 / 2} \quad$ and $\quad \boldsymbol{\sigma} \cdot \boldsymbol{\tau}=\sigma_{i j} \tau_{i j}, \quad|\boldsymbol{\tau}|=(\boldsymbol{\tau} \cdot \boldsymbol{\tau})^{1 / 2}$.

We shall adopt the usual notations for normal and tangential components of displacement vector and stress: $\boldsymbol{u}=u_{\nu} \boldsymbol{\nu}+\boldsymbol{u}_{\tau}, u_{\nu}=u_{i} \nu_{i}, \boldsymbol{\sigma} \boldsymbol{\nu}=\sigma_{\nu} \boldsymbol{\nu}+$ $\boldsymbol{\sigma}_{\tau}, \sigma_{\nu}=\sigma_{i j} \nu_{i} \nu_{j}$ 
We write the equations of motion and Maxwell-Gauss's law as follows:

$$
\begin{gathered}
\rho \ddot{\boldsymbol{u}}-\operatorname{Div} \boldsymbol{\sigma}=\boldsymbol{f}_{0} \quad \text { in } \quad \Omega \times(0, T), \\
\operatorname{div} \boldsymbol{D}=q_{0} \quad \text { in } \quad \Omega \times(0, T),
\end{gathered}
$$

where $\boldsymbol{\sigma}$ is the stress tensor, $\boldsymbol{u}$ denotes the displacement vector, $\boldsymbol{D}$ is the dielectric displacement vector, $\rho$ denotes the density of mass, $\boldsymbol{f}_{0}$ represents the density of body forces, $q_{0}$ is the volume density of free electric charges.

Notice also that Div and div represent the divergence operators for tensor and vector valued functions, that is

$$
\operatorname{Div} \boldsymbol{\sigma}=\left(\sigma_{i j, j}\right), \quad \operatorname{div} \boldsymbol{D}=D_{i, i} .
$$

For linear piezoelectric material we have the following constitutive relations

$$
\begin{gathered}
\boldsymbol{\sigma}=\mathcal{A} \varepsilon(\boldsymbol{u})+\mathcal{C} \varepsilon(\dot{\boldsymbol{u}})-\mathcal{E}^{*} \boldsymbol{E}(\varphi), \\
\boldsymbol{D}=\mathcal{E} \varepsilon(\boldsymbol{u})+\boldsymbol{\beta} \boldsymbol{E}(\varphi),
\end{gathered}
$$

where $\mathcal{A}=\left(a_{i j k l}\right), \mathcal{C}=\left(c_{i j k l}\right), \mathcal{E}=\left(e_{i j k}\right)$ and $\boldsymbol{\beta}=\left(\beta_{i j}\right)$ are respectively the (fourth-order) elasticity tensor, the (fourth-order) viscosity tensor, the (thirdorder) piezoelectric tensor and the electric permittivity tensor. $\boldsymbol{\varepsilon}$ and $\boldsymbol{E}$ are respectively, the linearized strain tensor and the electric intensity vector. We use $\mathcal{E}^{*}$ to denote the transpose of the tensor $\mathcal{E}$ given by:

$$
\mathcal{E} \boldsymbol{\sigma} \cdot \boldsymbol{v}=\boldsymbol{\sigma} \cdot \mathcal{E}^{*} \boldsymbol{v}, \quad \forall \boldsymbol{\sigma} \in \mathbb{S}^{d}, \quad \forall \boldsymbol{v} \in \mathbb{R}^{d} .
$$

The linearized strain tensor $\varepsilon$ and the electric intensity vector $\boldsymbol{E}$ are related to the displacement vector $\boldsymbol{u}$ and the electric potential $\varphi$ through the following:

$$
\boldsymbol{\varepsilon}(\boldsymbol{u})=\frac{1}{2}\left[\nabla \boldsymbol{u}+(\nabla \boldsymbol{u})^{*}\right] \text { and } \boldsymbol{E}=-\nabla \varphi .
$$

Next, we need to prescribe the mechanical and electrical boundary conditions. To this end, we consider first a partition of $\Gamma$ into three measurable parts $\Gamma_{1}, \Gamma_{2}, \Gamma_{3}$ such that meas $\left(\Gamma_{1}\right)>0$. We assume that the body is clamped on $\Gamma_{1}$ and surfaces tractions of density $\boldsymbol{f}_{2}$ act on $\Gamma_{2}$ that is:

$$
\boldsymbol{u}=\mathbf{0} \quad \text { on } \quad \Gamma_{1} \times(0, T) \text { and } \boldsymbol{\sigma} \boldsymbol{\nu}=\boldsymbol{f}_{2} \quad \text { on } \quad \Gamma_{2} \times(0, T) .
$$

On $\Gamma_{3}$ the body can arrive in frictional contact with an obstacle, the so-called foundation and the contact is given by:

$$
\begin{cases}\text { (a) } \sigma_{\nu}=-S, S \geq 0 & \text { on } \Gamma_{3} \times(0, T), \\ \text { (b) } \boldsymbol{\sigma}_{\tau}=-S \mu\left(\left|\boldsymbol{u}_{\tau}\right|\right) \frac{\dot{\boldsymbol{u}}_{\tau}}{\left|\dot{\boldsymbol{u}}_{\tau}\right|} & \text { if } \dot{\boldsymbol{u}}_{\tau} \neq \mathbf{0} \quad \text { on } \quad \Gamma_{3} \times(0, T), \\ \text { (c) }\left|\boldsymbol{\sigma}_{\tau}\right| \leq S \mu\left(\left|\boldsymbol{u}_{\tau}\right|\right) & \text { if } \dot{\boldsymbol{u}}_{\tau}=\mathbf{0} \quad \text { on } \quad \Gamma_{3} \times(0, T) .\end{cases}
$$

These equations represent a simplified version of Coulomb's law of dry friction in which the coefficient of friction depends on the slip, i.e., the contact surface 
is given and we impose a non-positive normal stress $-S$. Relations (2.6) assert that the tangential stress is bounded by the normal stress multiplied by the value of the friction coefficient $\mu\left(\left|\boldsymbol{u}_{\tau}\right|\right)$. If such a limit is not attained, sliding does not occur.

To describe the electric boundary conditions we assume that $\Gamma$ is divided into two disjoints measurable parts $\Gamma_{a}$ and $\Gamma_{b}$ such that meas $\left(\Gamma_{a}\right)>0$. We also assume, for simplicity, that the electrical potential vanishes on $\Gamma_{a}$ and the electric charge $q_{2}$ is prescribed on $\Gamma_{b}$ :

$$
\varphi=0 \quad \text { on } \quad \Gamma_{a} \times(0, T) \text { and } \boldsymbol{D} \cdot \boldsymbol{\nu}=q_{2} \quad \text { on } \quad \Gamma_{b} \times(0, T) .
$$

Finally, we prescribe the initials conditions that is

$$
\boldsymbol{u}=\boldsymbol{u}_{0}, \quad \dot{\boldsymbol{u}}(0)=\boldsymbol{v}_{0} \quad \text { in } \Omega .
$$

Our main goal here is to prove uniqueness and existence of weak solution to the following problem.

Problem P. Find a displacements field $\boldsymbol{u}: \Omega \times[0, T] \rightarrow \mathbb{R}^{d}$ and an electric potential field $\varphi: \Omega \times[0, T] \rightarrow \mathbb{R}$ such that (2.1)-(2.8) hold.

\section{WEAK FORMULATION AND MAIN RESUlT}

In this section we derive a weak formulation of the problem $P$ and investigate its solvability. Everywhere in what follows we use the classical notation for the $L^{p}$ and Sobolev spaces associated to $\Omega$ and $\Gamma$. Let $X$ be a Banach space, $T$ a positive real number then for $k=1,2, \ldots$, we also use the classical notations for the $W^{k, p}(0, T ; X)$. We also introduce the following functional spaces: $\mathbf{L}^{2}(\Omega)=\left[L^{2}(\Omega)\right]^{d}, \quad \mathbf{L}^{2}\left(\Gamma_{i}\right)=\left[L^{2}\left(\Gamma_{i}\right)\right]^{d}$, for $i=1,2,3, \mathbf{H}^{1}(\Omega)=\left[H^{1}(\Omega)\right]^{d}$.

We suppose that density $\rho$ satisfy

$$
\rho \in L^{\infty}(\Omega), \exists \rho_{0}>0 \text { such that } \rho(\boldsymbol{x}) \geq \rho_{0}>0, \text { a.e. } \boldsymbol{x} \in \Omega,
$$

and introduce over $\mathbf{L}^{2}(\Omega)$ the inner product

$$
(\boldsymbol{u}, \boldsymbol{v})_{\mathbf{L}^{2}(\Omega)}:=\int_{\Omega} \boldsymbol{u} \cdot \boldsymbol{v} d x, \forall \boldsymbol{u}, \boldsymbol{v} \in \mathbf{L}^{2}(\Omega)
$$

and denote by $|\cdot|_{\mathbf{L}^{2}(\Omega)}$ the associated norm. We denote by $H$ the space $\mathbf{L}^{2}(\Omega)$ equipped with the inner product $(\cdot, \cdot)_{H}$ defined by

$$
(\boldsymbol{u}, \boldsymbol{v})_{H}:=\int_{\Omega} \rho \boldsymbol{u} \cdot \boldsymbol{v} d x, \forall \boldsymbol{u}, \boldsymbol{v} \in H
$$

and we denote the associated norm by $|\cdot|_{H}$, i.e,

$$
|\boldsymbol{v}|_{H}=\left(\int_{\Omega} \rho|\boldsymbol{v}|^{2} d x\right)^{\frac{1}{2}} .
$$

Thus, the norms $|\cdot|_{\mathbf{L}^{2}(\Omega)}$ and $|\cdot|_{H}$ are equivalent over $\mathbf{L}^{2}(\Omega)$.

Moreover, keeping in mind (2.5), we introduce the following space:

$$
V=\left\{\boldsymbol{v} \in \mathbf{H}^{1}(\Omega) ; \boldsymbol{v}=\mathbf{0} \text { a.e. on } \Gamma_{1}\right\} .
$$


Next, for the stress field, we use the space

$$
Q=\left\{\boldsymbol{\tau}=\left(\tau_{i j}\right) \mid \tau_{i j}=\tau_{j i} \in L^{2}(\Omega)\right\}
$$

which is real Hilbert space endowed with the inner product

$$
(\boldsymbol{\sigma}, \boldsymbol{\tau})_{Q}=\int_{\Omega} \sigma_{i j} \tau_{i j} d x
$$

and the associated norm $\|\cdot\|_{Q}$.

It follows from Korn's inequality that the bilinear form defined over the space $V$ by

$$
(\boldsymbol{u}, \boldsymbol{v})_{V}=(\varepsilon(\boldsymbol{u}), \boldsymbol{\varepsilon}(\boldsymbol{v}))_{Q}
$$

is an inner product, the associated norm is denoted by $\|\cdot\|$. Then $\left(V,(\cdot, \cdot)_{V}\right)$ is a real Hilbert space. Since $V$ is dense in $H$, we identify $H$ and its dual and we write $V \hookrightarrow H \equiv(H)^{\prime} \hookrightarrow V^{\prime}$. We use $\langle\cdot, \cdot\rangle_{V^{\prime}, V}$ to denote the duality between $V^{\prime}$ and $V$. Moreover, by the Sobolev trace theorem, there exists a positive constant $c_{1}$ which depends on $\Omega, \Gamma_{1}$ and $\Gamma_{3}$ such that

$$
\|\boldsymbol{v}\|_{L^{2}\left(\Gamma_{3}\right)^{d}} \leq c_{1}\|\boldsymbol{v}\| \quad \forall \boldsymbol{v} \in V .
$$

Everywhere below, we denote by $c_{i}, C_{i}$ or $C$ various positive constants which are independent of time and whose value may change from line to line.

Next, for the electric potential we use the functional space

$$
W=\left\{\psi \in H^{1}(\Omega) ; \psi=0 \text { on } \Gamma_{a}\right\},
$$

which is real Hilbert space with the inner product $(\varphi, \psi)_{W}=(\varphi, \psi)_{H^{1}(\Omega)}$ and the associated norm $\|\cdot\|_{W}$. Since $W$ is dense in $L^{2}(\Omega)$, we identify $L^{2}(\Omega)$ and $\left(L^{2}(\Omega)\right)^{\prime}$ and write $W \hookrightarrow L^{2}(\Omega) \equiv\left(L^{2}(\Omega)\right)^{\prime} \hookrightarrow W^{\prime}$. We use $\langle\cdot, \cdot\rangle_{W^{\prime}, W}$ to denote the duality between $W^{\prime}$ and $W$. Moreover, by the Sobolev trace theorem, there exists a constant $c_{2}$, depending only on $\Omega, \Gamma_{a}$ and $\Gamma_{3}$, such that

$$
\|\zeta\|_{L^{2}\left(\Gamma_{3}\right)} \leq c_{2}\|\zeta\|_{W} \quad \forall \zeta \in W .
$$

We suppose that the friction coefficient $\mu: \Gamma_{3} \times \mathbb{R}_{+} \mapsto \mathbb{R}_{+}$is differentiable with respect to the second variable and there exist $M_{1}, \mu_{0}>0$ such that

$$
\begin{gathered}
0 \leq \mu(\boldsymbol{x}, \alpha) \leq \mu_{0}, \text { a.e. } \boldsymbol{x} \in \Gamma_{3}, \forall \alpha \in \mathbb{R}_{+}, \\
\left|\partial_{\alpha} \mu(\boldsymbol{x}, \alpha)\right| \leq M_{1}, \text { a.e. } \boldsymbol{x} \in \Gamma_{3}, \forall \alpha \in \mathbb{R}_{+},
\end{gathered}
$$

and the functions $\boldsymbol{x} \mapsto \mu(\boldsymbol{x}, \alpha)$ and $\boldsymbol{x} \mapsto \partial_{\alpha} \mu(\boldsymbol{x}, \alpha)$ are measurable for all $\alpha \in \mathbb{R}_{+}$.

For the normal stress we suppose that

$$
S(s) \geq 0 \text { for all } s \in \Gamma_{3} \text { and } S \in L^{\infty}\left(\Gamma_{3}\right) .
$$

Assume that the body forces and the surface tractions satisfy:

$$
\boldsymbol{f}_{0} \in W^{1,2}\left(0, T ; \mathbf{L}^{2}(\Omega)\right) \text { and } \boldsymbol{f}_{2} \in W^{1,2}\left(0, T ; \mathbf{L}^{2}\left(\Gamma_{2}\right)\right),
$$


and the volume and surface densities of the electric charges satisfy:

$$
q_{0} \in W^{1,2}\left(0, T ; L^{2}(\Omega)\right) \text { and } q_{2} \in W^{1,2}\left(0, T ; L^{2}\left(\Gamma_{b}\right)\right) .
$$

The initial conditions will be supposed as follows:

$$
\boldsymbol{u}_{0}, \boldsymbol{v}_{0} \in V \text {. }
$$

Keeping in mind assumptions (3.7)-(3.8), it follows that the there exists $\boldsymbol{f} \in$ $W^{1,2}\left(0, T ; V^{\prime}\right)$ and $q \in W^{1,2}\left(0, T ; W^{\prime}\right)$ such that next integrals are welldefined

$$
\begin{gathered}
\langle\boldsymbol{f}(t), \boldsymbol{v}\rangle_{V^{\prime}, V}=\int_{\Omega} \boldsymbol{f}_{0}(t) \cdot \boldsymbol{v} d x+\int_{\Gamma_{2}} \boldsymbol{f}_{2}(t) \cdot \boldsymbol{v} d s-\int_{\Gamma_{3}} S v_{\nu} d s, \forall \boldsymbol{v} \in V, \\
\langle q(t), \psi\rangle_{W^{\prime}, W}=\int_{\Omega} q_{0}(t) \psi d x-\int_{\Gamma_{b}} q_{2}(t) \psi d s, \quad \forall \psi \in W .
\end{gathered}
$$

$$
j(\boldsymbol{u}, \boldsymbol{v})=\int_{\Gamma_{3}} S \mu\left(\left|\boldsymbol{u}_{\tau}\right|\right)\left|\boldsymbol{v}_{\tau}\right| d s, \forall \boldsymbol{u}, \boldsymbol{v} \in V .
$$

Finally, we denote by $a: V \times V \longrightarrow \mathbb{R}, c: V \times V \longrightarrow \mathbb{R}, b: W \times W \longrightarrow \mathbb{R}$ and $e: V \times W \longrightarrow \mathbb{R}$ the following bilinear applications:

$$
a(\boldsymbol{u}, \boldsymbol{v}):=(\mathcal{A} \varepsilon(\boldsymbol{u}), \boldsymbol{\varepsilon}(\boldsymbol{v}))_{Q}, \quad c(\boldsymbol{u}, \boldsymbol{v}):=(\mathcal{C} \varepsilon(\boldsymbol{u}), \boldsymbol{\varepsilon}(\boldsymbol{v}))_{Q},
$$

$b(\varphi, \psi):=(\boldsymbol{\beta} \nabla \varphi, \nabla \psi)_{\mathbf{L}^{2}(\Omega)}, \quad e(\boldsymbol{u}, \varphi):=(\mathcal{E} \varepsilon(\boldsymbol{u}), \nabla \varphi)_{\mathbf{L}^{2}(\Omega)}=\left(\mathcal{E}^{*} \nabla \varphi, \boldsymbol{\varepsilon}(\boldsymbol{u})\right)_{Q}$. Suppose that $\mathcal{A}, \mathcal{C}, \boldsymbol{\beta}$ and $\mathcal{E}$ satisfy the usual properties of symmetry, boundedness and ellipticity:

$$
\begin{gathered}
a_{i j k l}=a_{k l i j}=a_{j i k l} \in L^{\infty}(\Omega), \quad c_{i j k l}=c_{k l i j}=c_{j i k l} \in L^{\infty}(\Omega), \\
\beta_{i j}=\beta_{j i} \in L^{\infty}(\Omega), \quad e_{i j k}=e_{i k j} \in L^{\infty}(\Omega),
\end{gathered}
$$

and there exists $m>0$ such that

$$
\begin{gathered}
a_{i j k l} \varepsilon_{i j} \varepsilon_{k l} \geq m|\varepsilon|^{2}, \quad \forall \varepsilon \in \mathbb{S}^{d}, \quad c_{i j k l} \varepsilon_{i j} \varepsilon_{k l} \geq m|\varepsilon|^{2}, \quad \forall \varepsilon \in \mathbb{S}^{d}, \\
\beta_{i j} E_{i} E_{j} \geq m|\boldsymbol{E}|^{2}, \quad \forall \boldsymbol{E} \in \mathbb{R}^{d} .
\end{gathered}
$$

It is easy to see that $a$ and $c$ are continuous, $V$-elliptic forms and $b$ is continuous and $W$-elliptic form and $e$ is continuous on $V \times W$. The coercivity of the form $c(\cdot, \cdot)$ implies that

$$
\exists D>0 \text { such that } c(\boldsymbol{v}, \boldsymbol{v}) \geq D\|\boldsymbol{v}\|^{2}, \forall \boldsymbol{v} \in V .
$$

Now, using integration by parts, it is straightforward to see that if $(\boldsymbol{u}, \varphi)$ are sufficiently regular functions satisfying (2.1)-(2.8) then, for all $t \in[0, T]$, we have

$$
\begin{aligned}
& (\ddot{\boldsymbol{u}}(t), \boldsymbol{w})_{H}+(\boldsymbol{\sigma}(t), \boldsymbol{\varepsilon}(\boldsymbol{w}))_{Q} \\
& \quad=\int_{\Gamma_{3}} \boldsymbol{\sigma}_{\tau}(t) \cdot \boldsymbol{w}_{\tau} d s+\langle\boldsymbol{f}(t), \boldsymbol{w}\rangle_{V^{\prime}, V}, \forall \boldsymbol{w} \in V,
\end{aligned}
$$




$$
(\boldsymbol{D}(t), \nabla \psi)_{\mathbf{L}^{2}(\Omega)}+\langle q(t), \psi\rangle_{W^{\prime}, W}=0, \forall \psi \in W .
$$

Keeping in mind (2.6) we find

$\int_{\Gamma_{3}} \boldsymbol{\sigma}_{\tau}(t) \cdot\left(\boldsymbol{v}_{\tau}-\dot{\boldsymbol{u}}_{\tau}(t)\right) d s+j(\boldsymbol{u}(t), \boldsymbol{v})-j(\boldsymbol{u}(t), \dot{\boldsymbol{u}}(t)) \geq 0, \forall \boldsymbol{v} \in V, t \in[0, T]$.

Therefore, by taking $\boldsymbol{w}=\boldsymbol{v}-\dot{\boldsymbol{u}}(t)$ in (3.14) we have:

$$
\begin{gathered}
(\ddot{\boldsymbol{u}}(t), \boldsymbol{v}-\dot{\boldsymbol{u}}(t))_{H}+\left(\boldsymbol{\sigma}(t), \boldsymbol{\varepsilon}(\boldsymbol{v})-\boldsymbol{\varepsilon}(\dot{\boldsymbol{u}}(t))_{Q}+j(\boldsymbol{u}(t), \boldsymbol{v})-j(\boldsymbol{u}(t), \dot{\boldsymbol{u}}(t))\right. \\
\geq\langle\boldsymbol{f}(t), \boldsymbol{v}-\dot{\boldsymbol{u}}(t)\rangle_{V^{\prime}, V}, \forall \boldsymbol{v} \in V, t \in[0, T] .
\end{gathered}
$$

We plug now (2.3) in (3.16), (2.4) in (3.15), use the notation $\boldsymbol{E}=-\nabla \varphi$ and the initial condition (2.8) to derive the following variational formulation of problem $P$, in the terms of displacement and electric potential fields.

Problem $\mathrm{P}_{V}$. Find the displacement field $\boldsymbol{u}:[0, T] \rightarrow V$ and the electric potential $\varphi:[0, T] \rightarrow W$ such that

$$
\begin{gathered}
(\ddot{\boldsymbol{u}}(t), \boldsymbol{v}-\dot{\boldsymbol{u}}(t))_{H}+a(\boldsymbol{u}(t), \boldsymbol{v}-\dot{\boldsymbol{u}}(t))+c(\dot{\boldsymbol{u}}(t), \boldsymbol{v}-\dot{\boldsymbol{u}}(t)) \\
+e(\boldsymbol{v}-\dot{\boldsymbol{u}}(t), \varphi(t))+j(\boldsymbol{u}(t), \boldsymbol{v})-j(\boldsymbol{u}(t), \dot{\boldsymbol{u}}(t)) \\
\geq\langle\boldsymbol{f}(t), \boldsymbol{v}-\dot{\boldsymbol{u}}(t)\rangle_{V^{\prime}, V} \quad \forall \boldsymbol{v} \in V, t \in[0, T], \\
b(\varphi(t), \psi)-e(\boldsymbol{u}(t)), \psi)=\langle q(t), \psi\rangle_{W^{\prime}, W} \forall \psi \in W, t \in[0, T], \\
\boldsymbol{u}(0)=\boldsymbol{u}_{0}, \quad \dot{\boldsymbol{u}}(0)=\boldsymbol{v}_{0} .
\end{gathered}
$$

The main results of this section is the following:

Theorem 3.1. Let $d=2,3$. Assume that (3.1)-(3.9). Then under the previous assumptions on $\mathcal{A}, \mathcal{C}, \boldsymbol{\beta}$ and $\mathcal{E}$, there exists a unique solution $(\boldsymbol{u}, \varphi)$ of Problem $P_{V}$ such that

$$
\boldsymbol{u} \in W^{1, \infty}(0, T ; V) \cap W^{2,2}(0, T ; H), \varphi \in W^{1,2}(0, T ; W) .
$$

\section{Proof of Theorem 3.1}

The proof of Theorem 3.1 will be carried out in several steps.

UNIQUeNESS. Let us consider two solutions $\left(\boldsymbol{u}_{1}, \varphi_{1}\right),\left(\boldsymbol{u}_{2}, \varphi_{2}\right)$ to problem $P_{V}$ with the regularity $(3.20)$ and write $\boldsymbol{w}(t)=\boldsymbol{u}_{2}(t)-\boldsymbol{u}_{1}(t)$ and $\phi(t)=$ $\varphi_{2}(t)-\varphi_{1}(t)$. If we write the variational inequality (3.17) successively for $\left(\boldsymbol{u}_{1}, \varphi_{1}\right)$ and $\left(\boldsymbol{u}_{2}, \varphi_{2}\right)$, taking $\boldsymbol{v}=\dot{\boldsymbol{u}}_{2}(t)$ in the first inequality and $\boldsymbol{v}=\dot{\boldsymbol{u}}_{1}(t)$ in the second one, and add the resulting inequalities, we obtain

$$
\begin{aligned}
& (\ddot{\boldsymbol{w}}(t), \dot{\boldsymbol{w}}(t))_{H}+a(\boldsymbol{w}(t), \dot{\boldsymbol{w}}(t))+c(\dot{\boldsymbol{w}}(t), \dot{\boldsymbol{w}}(t))+e(\dot{\boldsymbol{w}}(t), \phi(t)) \\
& +j\left(\boldsymbol{u}_{1}(t), \dot{\boldsymbol{u}}_{1}(t)\right)+j\left(\boldsymbol{u}_{2}(t), \dot{\boldsymbol{u}}_{2}(t)\right)-j\left(\boldsymbol{u}_{1}(t), \dot{\boldsymbol{u}}_{2}(t)\right) \\
& -j\left(\boldsymbol{u}_{2}(t), \dot{\boldsymbol{u}}_{1}(t)\right) \leq 0, \forall t \in(0, T) .
\end{aligned}
$$


We can easily deduce from variational equality (3.18) that

$$
e(\boldsymbol{w}(t), \psi)=b(\phi(t), \psi), \forall t \in(0, T), \forall \psi \in W,
$$

which implies that

$$
e(\dot{\boldsymbol{w}}(t), \phi(t))=b(\dot{\phi}(t), \phi(t)), \forall t \in(0, T) .
$$

Having in mind (3.12), we obtain

(4.4)

$$
\begin{aligned}
& \left|j\left(\boldsymbol{u}_{1}(t), \dot{\boldsymbol{u}}_{1}(t)\right)+j\left(\boldsymbol{u}_{2}(t), \dot{\boldsymbol{u}}_{2}(t)\right)-j\left(\boldsymbol{u}_{1}(t), \dot{\boldsymbol{u}}_{2}(t)\right)-j\left(\boldsymbol{u}_{2}(t), \dot{\boldsymbol{u}}_{1}(t)\right)\right| \\
& \quad=\left|\int_{\Gamma_{3}} S(\boldsymbol{s})\left[\left(\mu\left(\boldsymbol{s},\left|\boldsymbol{u}_{1 \tau}(t)\right|\right)-\mu\left(\boldsymbol{s},\left|\boldsymbol{u}_{2 \tau}(t)\right|\right)\right)\left(\left|\dot{\boldsymbol{u}}_{1 \tau}(\boldsymbol{s}, t)\right|-\left|\dot{\boldsymbol{u}}_{2 \tau}(\boldsymbol{s}, t)\right|\right)\right] d s\right| \\
& \quad \leq c_{3} \int_{\Gamma_{3}}\left|\boldsymbol{u}_{1 \tau}(\boldsymbol{s}, t)-\boldsymbol{u}_{2 \tau}(\boldsymbol{s}, t)\right|\left|\dot{\boldsymbol{u}}_{1 \tau}(\boldsymbol{s}, t)-\dot{\boldsymbol{u}}_{2 \tau}(\boldsymbol{s}, t)\right| d s \\
& \quad \leq c_{4}\left\|\boldsymbol{u}_{1}(t)-\boldsymbol{u}_{2}(t)\right\|\left\|\dot{\boldsymbol{u}}_{1}(t)-\dot{\boldsymbol{u}}_{2}(t)\right\|
\end{aligned}
$$

where $c_{3}=M_{1}\|S\|_{L^{\infty}\left(\Gamma_{3}\right)}$ and $c_{4}=c_{3} \times c_{1}^{2}=M_{1}\|S\|_{L^{\infty}\left(\Gamma_{3}\right)} c_{1}^{2}$ which $M_{1}$ and $c_{1}$ are the constants defined respectively in (3.5) and (3.2).

Then, from (4.1), (4.3) and (4.4) we obtain

$$
\begin{aligned}
& \frac{1}{2} \frac{d}{d t}\left(|\dot{\boldsymbol{w}}(t)|_{H}^{2}+a(\boldsymbol{w}(t), \boldsymbol{w}(t))+b(\phi(t), \phi(t))\right)+c(\dot{\boldsymbol{w}}(t), \dot{\boldsymbol{w}}(t)) \\
& \leq c_{4}\|\boldsymbol{w}(t)\| \times\|\dot{\boldsymbol{w}}(t)\|, \forall t \in(0, T) .
\end{aligned}
$$

Using (4.2) with $\psi=\phi(0)$ and $t=0$; and coercivity of the bilinear form $b(\cdot, \cdot)$; we get $\phi(0)=0$. Since $\boldsymbol{w}(0)=\dot{\boldsymbol{w}}(0)=\mathbf{0}, \phi(0)=0$, if we integrate the above inequality from 0 to $t$, we obtain by using Young inequality

$$
\begin{gathered}
\frac{1}{2}\left(|\dot{\boldsymbol{w}}(t)|_{H}^{2}+a(\boldsymbol{w}(t), \boldsymbol{w}(t))+b(\phi(t), \phi(t))\right)+\int_{0}^{t} c(\dot{\boldsymbol{w}}(\theta), \dot{\boldsymbol{w}}(\theta)) d \theta \\
\leq \int_{0}^{t}\left(\epsilon\|\dot{\boldsymbol{w}}(\theta)\|^{2}+C_{\epsilon}\|\boldsymbol{w}(\theta)\|^{2}\right) d \theta, \forall t \in(0, T)
\end{gathered}
$$

By choosing $\epsilon=\frac{D}{2}$ (where $D$ is given in (3.13)) and coercivity of the bilinear forms $a, b$ we deduce

$$
\begin{aligned}
& \left(|\dot{\boldsymbol{w}}(t)|_{H}^{2}+\|\boldsymbol{w}(t)\|^{2}+\|\phi(t)\|_{W}^{2}\right)+\int_{0}^{t}\|\dot{\boldsymbol{w}}(\theta)\|^{2} d \theta \\
& \quad \leq C \int_{0}^{t}\left(|\dot{\boldsymbol{w}}(\theta)|_{H}^{2}+\|\boldsymbol{w}(\theta)\|^{2}+\|\phi(\theta)\|_{W}^{2}\right) d \theta, \forall t \in(0, T) .
\end{aligned}
$$

The Gronwall's Lemma allows us to deduce from the above inequality that $\boldsymbol{w}(t)=\mathbf{0}$ and $\phi(t)=0, \forall t \in[0, T]$. This concludes the proof of uniqueness.

EXISTENCE. Firstly, by using Riesz's representation theorem we find the operators $B: W \rightarrow W^{\prime}, \Pi: V \rightarrow W^{\prime}$ and $\Pi^{*}: W \rightarrow V^{\prime}$ defined by:

$$
\langle B \varphi, \psi\rangle_{W^{\prime} W}=b(\varphi, \psi), \forall \psi \in W
$$




$$
\begin{gathered}
\langle\Pi \boldsymbol{v}, \psi\rangle_{W^{\prime}, W}=(\mathcal{E} \varepsilon(\boldsymbol{v}), \nabla \psi)_{L^{2}(\Omega)^{d}}=e(\boldsymbol{v}, \psi), \quad \forall \psi \in W ; \\
\left\langle\Pi^{*} \varphi, \boldsymbol{v}\right\rangle_{V^{\prime}, V}=\left(\mathcal{E}^{*} \nabla \varphi, \varepsilon(\boldsymbol{v})\right)_{Q}=e(\boldsymbol{v}, \varphi), \quad \forall \boldsymbol{v} \in V ;
\end{gathered}
$$

where $\Pi^{*}$ is the adjoint of $\Pi$.

Now, we prove the following equivalence result.

LEMma 4.1. The couple $(\boldsymbol{u}, \varphi)$ is a solution to problem $P_{V}$ if only if

$$
\begin{gathered}
(\ddot{\boldsymbol{u}}(t), \boldsymbol{v}-\dot{\boldsymbol{u}}(t))_{H}+\widetilde{a}(\boldsymbol{u}(t), \boldsymbol{v}-\dot{\boldsymbol{u}}(t))+c(\dot{\boldsymbol{u}}(t), \boldsymbol{v}-\dot{\boldsymbol{u}}(t)) \\
+j(\boldsymbol{u}(t), \boldsymbol{v})-j(\boldsymbol{u}(t), \dot{\boldsymbol{u}}(t)) \geq\langle\widetilde{\boldsymbol{f}}(t), \boldsymbol{v}-\dot{\boldsymbol{u}}(t)\rangle_{V^{\prime}, V}, \forall \boldsymbol{v} \in V \\
\boldsymbol{u}(0)=\boldsymbol{u}_{0}, \quad \dot{\boldsymbol{u}}(0)=\boldsymbol{v}_{0},
\end{gathered}
$$

where $\widetilde{a}(\boldsymbol{u}, \boldsymbol{v}):=a(\boldsymbol{u}, \boldsymbol{v})+\left\langle\Pi^{*} B^{-1} \Pi \boldsymbol{u}, \boldsymbol{v}\right\rangle_{V^{\prime}, V}$ and $\widetilde{\boldsymbol{f}}(t):=\boldsymbol{f}(t)-$ $\Pi^{*} B^{-1} q(t)$.

Proof. Let $(\boldsymbol{u}, \varphi)$ be a solution of $P_{V}$. We solve the equation (3.18) on $\boldsymbol{u}(t)$. To this end, let $\boldsymbol{u} \in C(0, T ; V)$ and find $\varphi:[0, T] \rightarrow W$ such that

$$
b(\varphi(t), \psi)=e(\boldsymbol{u}(t), \psi)+\langle q(t), \psi\rangle_{W^{\prime}, W}, \forall \psi \in W, \forall t \in[0, T] .
$$

From the continuity of $e$ on $V \times W$, we remark that for all $t \in[0, T]$ the linear form: $\psi \mapsto e(\boldsymbol{u}(t)), \psi)+\langle q(t), \psi\rangle_{W^{\prime}, W}$, is continuous on $W$. Since $b(\cdot, \cdot)$ is symmetric continuous and $W$-elliptic, it follows that $B$ is positive-definite and self-adjoint operator and is invertible such that $B^{-1}$ is linear continuous from $W^{\prime}$ to $W$. Using the Lax-Milgram theorem, we conclude that the equation (4.14) has unique solution $\varphi \in C(0, T ; W)$ which has an explicit form (4.13).

Now, we deduce that if $\boldsymbol{u}:[0, T] \rightarrow V$ is given and $\varphi:[0, T] \rightarrow W$ satisfies (4.13) we have

$$
e(\boldsymbol{w}, \varphi(t))=\left\langle\Pi^{*} B^{-1} \Pi \boldsymbol{u}(t)+\Pi^{*} B^{-1} q(t), \boldsymbol{w}\right\rangle_{V^{\prime}, V}, \forall \boldsymbol{w} \in V .
$$

Thus, by inserting the equation (4.15) with $\boldsymbol{w}=\boldsymbol{v}-\dot{\boldsymbol{u}}(t)$ in (3.17), we can deduce easily that the variational problem $P_{V}$ is equivalent to the problem (4.11)-(4.13) introduced in Lemma 4.1.

Let denote $a_{0}(\boldsymbol{u}, \boldsymbol{v}):=\left\langle\Pi^{*} B^{-1} \Pi \boldsymbol{u}, \boldsymbol{v}\right\rangle_{V^{\prime}, V}$, we observe that $a_{0}(\cdot, \cdot)$ is bilinear, symmetric continuous on $V$ and there exists $\alpha>0$ such that $a_{0}(\boldsymbol{v}, \boldsymbol{v}) \geq \alpha\|\Pi \boldsymbol{v}\|_{W^{\prime}}^{2} \geq 0$ for all $\boldsymbol{v}$ in $V$. Thus, we conclude that $\widetilde{a}$ is bilinear, symmetric, continuous and $V$ - elliptic.

Keeping in mind that $q \in W^{1,2}\left(0, T ; W^{\prime}\right)$ and properties of the operators $\Pi^{*}, B^{-1}$ which are linear continuous in their correspondent spaces, we deduce that $\widetilde{q}:[0, T] \rightarrow V^{\prime}$ defined by $\widetilde{q}(t):=-\Pi^{*} B^{-1} q(t)$ satisfies $\widetilde{q} \in W^{1,2}\left(0, T ; V^{\prime}\right)$ and therefore the function $\widetilde{\boldsymbol{f}} \in W^{1,2}\left(0, T ; V^{\prime}\right)$.

Now, we solve the transformed problem (4.11)-(4.12) on $\boldsymbol{u}$ as in [3] and [6]. We will use the regularized method followed by the Faedo-Galerkin's 
method and compactness and semicontinuity arguments. To this end let us consider $\left(\phi_{i}\right)_{i \in \mathbb{N}} \in V$ a sequence of linear independent functions such that $V=\bar{\cup}_{m=1}^{\infty} V^{m}$ where $V^{m}=\operatorname{Span}\left\{\phi_{1}, \phi_{2}, \ldots, \phi_{m}\right\}$. We shall suppose that $V^{m}$ is chosen such that for $m$ large enough we have $\boldsymbol{u}_{0}$ and $\boldsymbol{v}_{0}$ belong to $V^{m}$.

We also consider, for all $\epsilon>0$, the family of convex and differentiable functions $\psi_{\epsilon}: \mathbb{R}^{d} \rightarrow \mathbb{R}$ given by

$$
\psi_{\epsilon}(\boldsymbol{v}):=\sqrt{|\boldsymbol{v}|^{2}+\epsilon^{2}}-\epsilon, \forall \boldsymbol{v} \in \mathbb{R}^{d} .
$$

It is easy to show that such a family of functions satisfies:

$$
\begin{gathered}
0 \leq \psi_{\epsilon}(\boldsymbol{v}) \leq|\boldsymbol{v}|, \forall \boldsymbol{v} \in \mathbb{R}^{d}, \\
\left|\psi_{\epsilon}(\boldsymbol{v})-\right| \boldsymbol{v}|| \leq \epsilon, \forall \boldsymbol{v} \in \mathbb{R}^{d}, \\
\psi_{\epsilon}^{\prime}(\boldsymbol{v})(\boldsymbol{w})=\frac{\boldsymbol{v} \cdot \boldsymbol{w}}{\sqrt{|\boldsymbol{v}|^{2}+\epsilon^{2}}}, \forall \boldsymbol{v}, \boldsymbol{w} \in \mathbb{R}^{d} .
\end{gathered}
$$

From equation (4.18), we have

$$
\left|\psi_{\epsilon}^{\prime}(\boldsymbol{v})(\boldsymbol{w})\right| \leq|\boldsymbol{w}|, \forall \boldsymbol{v}, \boldsymbol{w} \in \mathbb{R}^{d} .
$$

We then define a family of regularized frictional functional $j_{\epsilon}: V \times V \rightarrow \mathbb{R}$ by

$$
j_{\epsilon}(\boldsymbol{u}, \boldsymbol{v})=\int_{\Gamma_{3}} S(\boldsymbol{s}) \mu\left(\boldsymbol{s},\left|\boldsymbol{u}_{\tau}(\boldsymbol{s})\right|\right) \psi_{\epsilon}\left(\boldsymbol{v}_{\tau}(\boldsymbol{s})\right) d s, \forall \boldsymbol{u}, \boldsymbol{v} \in V .
$$

The functionals $j_{\epsilon}$ are Gâteaux-differentiable with respect to the second argument $\boldsymbol{v}$ and represent an approximation of $j$, i.e., there exists a constant $C$ such that

$$
\left|j_{\epsilon}(\boldsymbol{u}, \boldsymbol{v})-j(\boldsymbol{u}, \boldsymbol{v})\right| \leq C \epsilon, \forall \boldsymbol{u}, \boldsymbol{v} \in V .
$$

We denote by $J_{\epsilon}: V \times V \rightarrow V^{\prime}$ the derivative of $j_{\epsilon}$ with respect to the second variable given by

$$
\left\langle J_{\epsilon}(\boldsymbol{u}, \boldsymbol{v}), \boldsymbol{z}\right\rangle_{V^{\prime}, V}=\int_{\Gamma_{3}} S(\boldsymbol{s}) \mu\left(\boldsymbol{s},\left|\boldsymbol{u}_{\tau}(\boldsymbol{s})\right|\right) \psi_{\epsilon}^{\prime}\left(\boldsymbol{v}_{\tau}(\boldsymbol{s})\right)\left(\boldsymbol{z}_{\tau}(\boldsymbol{s})\right) d s, \forall \boldsymbol{u}, \boldsymbol{v}, \boldsymbol{z} \in V .
$$

Now, we define the regularized problem associated to (4.11)-(4.12) in finitedimensional spaces: Find $\boldsymbol{u}_{\epsilon}^{m}:[0, T] \rightarrow V^{m}$ such that:

$$
\begin{gathered}
\left(\ddot{\boldsymbol{u}}_{\epsilon}^{m}(t), \boldsymbol{v}\right)_{H}+\widetilde{a}\left(\boldsymbol{u}_{\epsilon}^{m}(t), \boldsymbol{v}\right)+c\left(\dot{\boldsymbol{u}}_{\epsilon}^{m}(t), \boldsymbol{v}\right)+\left\langle J_{\epsilon}\left(\boldsymbol{u}_{\epsilon}^{m}(t), \dot{\boldsymbol{u}}_{\epsilon}^{m}(t)\right), \boldsymbol{v}\right\rangle_{V^{\prime}, V} \\
=\langle\widetilde{\boldsymbol{f}}(t), \boldsymbol{v}\rangle_{V^{\prime}, V}, \forall \boldsymbol{v} \in V, \forall t \in[0, T] . \\
\boldsymbol{u}_{\epsilon}^{m}(0)=\boldsymbol{u}_{0}, \quad \dot{\boldsymbol{u}}_{\epsilon}^{m}(0)=\boldsymbol{v}_{0} .
\end{gathered}
$$

Since $(\boldsymbol{u}, \boldsymbol{v}) \mapsto J_{\epsilon}(\boldsymbol{u}, \boldsymbol{v})$ is locally Lipschitz continuous on $V^{m} \times V^{m}$ we deduce that for all $\epsilon>0$ and $m \in \mathbb{N}$ the problem(4.23)-(4.24) has an unique maximal solution $\boldsymbol{u}_{\epsilon}^{m} \in C^{2}\left(0, T_{\epsilon}^{m} ; V^{m}\right)$. The a priori estimates of our regularized problem are given by the following lemma. 
Lemma 4.2. Under the assumptions of Theorem 3.1, there exists a unique solution $\boldsymbol{u}_{\epsilon}^{m}$ of Problem (4.23)- (4.24) such that for every $\epsilon>0, m \in \mathbb{N}$, we have

$$
\left\{\begin{array}{l}
\left\{\boldsymbol{u}_{\epsilon}^{m}\right\}_{m, \epsilon}, \quad\left\{\dot{\boldsymbol{u}}_{\epsilon}^{m}\right\}_{m, \epsilon} \text { are bounded in } L^{\infty}(0, T ; V), \\
\left\{\ddot{\boldsymbol{u}}_{\epsilon}^{m}\right\}_{m, \epsilon} \text { is bounded in } L^{2}(0, T ; H) .
\end{array}\right.
$$

Proof. First, let observe that in [3] and [6] the authors derive the above estimates for similar problem, with different functional $j$ by assuming that $\widetilde{\boldsymbol{f}} \in W^{1,2}\left(0, T ; V^{\prime}\right)$. Here, to establish the Estimates I, we only assume that $\widetilde{\boldsymbol{f}} \in L^{2}\left(0, T ; V^{\prime}\right)$.

The proof of estimates II follows the lines of [6]. We thus do not detail the proof in the present paper. We give briefly the outline of the proof:

Estimates $I$. Since $\left\langle J_{\epsilon}(\boldsymbol{u}, \boldsymbol{v}), \boldsymbol{v}\right\rangle_{V^{\prime}, V} \geq 0$ for all $\boldsymbol{u}, \boldsymbol{v} \in V$, if we take $\boldsymbol{v}=\dot{\boldsymbol{u}}_{\epsilon}^{m}$ in (4.23), we obtain

$$
\frac{1}{2} \frac{d}{d t}\left(\left|\dot{\boldsymbol{u}}_{\epsilon}^{m}(t)\right|_{H}^{2}+\widetilde{a}\left(\boldsymbol{u}_{\epsilon}^{m}(t), \boldsymbol{u}_{\epsilon}^{m}(t)\right)\right)+c\left(\dot{\boldsymbol{u}}_{\epsilon}^{m}(t), \dot{\boldsymbol{u}}_{\epsilon}^{m}(t)\right) \leq\left\langle\widetilde{\boldsymbol{f}}(t), \dot{\boldsymbol{u}}_{\epsilon}^{m}(t)\right\rangle_{V^{\prime}, V}
$$

Using the classical techniques, we can easily deduce that there exists $C>0$, which is independent of $\boldsymbol{u}_{0}, \boldsymbol{v}_{0}$ and $\widetilde{\boldsymbol{f}}$, such that: $\forall t \in\left[0, T_{\epsilon}^{m}\right]$

$$
\begin{aligned}
& \left\|\boldsymbol{u}_{\epsilon}^{m}(t)\right\|^{2}+\left|\dot{\boldsymbol{u}}_{\epsilon}^{m}(t)\right|_{H}^{2}+\int_{0}^{t}\left\|\dot{\boldsymbol{u}}_{\epsilon}^{m}(t)(\theta)\right\|^{2} d \theta \\
& \quad \leq C\left(\left\|\boldsymbol{u}_{0}\right\|^{2}+\left|\boldsymbol{v}_{0}\right|_{H}^{2}+\int_{0}^{t}\|\widetilde{\boldsymbol{f}}(\theta)\|_{V^{\prime}}^{2} d \theta\right), \quad \forall t \in\left[0, T_{\epsilon}^{m}\right] .
\end{aligned}
$$

So the unique solution of the approximate problem is global in time, i.e., $T_{\epsilon}^{m}=T$ and

$$
\left\{\begin{array}{l}
\left\{\boldsymbol{u}_{\epsilon}^{m}\right\}_{m, \epsilon} \text { is bounded in } L^{\infty}(0, T ; V), \\
\left\{\dot{\boldsymbol{u}}_{\epsilon}^{m}\right\}_{m, \epsilon} \text { is bounded in } L^{\infty}(0, T ; H) \cap L^{2}(0, T ; V) .
\end{array}\right.
$$

Estimates II. Setting $\boldsymbol{v}=\ddot{\boldsymbol{u}}_{\epsilon}^{m}(t)$ in (4.23) and using that $\frac{\partial}{\partial t}\left(\psi_{\epsilon}\left(\dot{\boldsymbol{u}}_{\tau}(\boldsymbol{s}, t)\right)=\right.$ $\left(\frac{\dot{\boldsymbol{u}}_{\tau} \cdot \ddot{\boldsymbol{u}}_{\tau}}{\left(\left|\dot{\boldsymbol{u}}_{\tau}\right|^{2}+\epsilon^{2}\right)^{\frac{1}{2}}}\right)(\boldsymbol{s}, t)$, we obtain

$$
\begin{aligned}
& \left|\ddot{\boldsymbol{u}}_{\epsilon}^{m}(t)\right|_{H}^{2}+\widetilde{a}\left(\boldsymbol{u}_{\epsilon}^{m}(t), \ddot{\boldsymbol{u}}_{\epsilon}^{m}(t)\right)+\frac{1}{2} \frac{d}{d t} c\left(\dot{\boldsymbol{u}}_{\epsilon}^{m}(t), \dot{\boldsymbol{u}}_{\epsilon}^{m}(t)\right) \\
& \quad+\int_{\Gamma_{3}} S(\boldsymbol{s}) \mu\left(\boldsymbol{s},\left|\boldsymbol{u}_{\tau}(\boldsymbol{s}, t)\right|\right) \frac{\partial}{\partial t} \psi_{\epsilon}\left(\dot{\boldsymbol{u}}_{\tau}(t)\right) d s=\left\langle\widetilde{\boldsymbol{f}}(t), \ddot{\boldsymbol{u}}_{\epsilon}^{m}(t)\right\rangle_{V^{\prime}, V}
\end{aligned}
$$


Now, using coercivity of the form $c(\cdot, \cdot)$, the last equality and integrating over $(0, t), 0 \leq t \leq T$, the equation (4.28) we get

$$
\begin{aligned}
\int_{0}^{t} \mid & \left.\ddot{\boldsymbol{u}}_{\epsilon}^{m}(\theta)\right|_{H} ^{2} d \theta+\frac{D}{2}\left\|\dot{\boldsymbol{u}}_{\epsilon}^{m}(t)\right\|^{2} \\
\leq & \frac{1}{2} c\left(\boldsymbol{v}_{0}, \boldsymbol{v}_{0}\right)-\int_{0}^{t} \widetilde{a}\left(\boldsymbol{u}_{\epsilon}^{m}(\theta), \ddot{\boldsymbol{u}}_{\epsilon}^{m}(\theta)\right) d \theta+\int_{0}^{t}\left\langle\widetilde{\boldsymbol{f}}(\theta), \ddot{\boldsymbol{u}}_{\epsilon}^{m}(\theta)\right\rangle_{V^{\prime}, V} d \theta \\
& \quad-\int_{0}^{t} \int_{\Gamma_{3}} S(\boldsymbol{s}) \mu\left(\boldsymbol{s},\left|\boldsymbol{u}_{\epsilon \tau}^{m}(\boldsymbol{s}, \theta)\right|\right) \frac{\partial}{\partial \theta} \psi_{\epsilon}\left(\dot{\boldsymbol{u}}_{\epsilon \tau}^{m}(\boldsymbol{s}, \theta)\right) d s d \theta
\end{aligned}
$$

Let us remark that we use here a simplified version of mechanical problem studied in [6] with operators which take into account the piezoelectrical effect. Now, adapting the proof of [6] to the present case, one can obtain the following estimates: there exists $C>0$ such that

$$
\left\|\dot{\boldsymbol{u}}_{\epsilon}^{m}(t)\right\|^{2}+\int_{0}^{T}\left|\ddot{\boldsymbol{u}}_{\epsilon}^{m}(t)\right|_{H}^{2} d t \leq C .
$$

Thus, from estimates I and estimates II, we get the estimates given in Lemma 4.2 .

PAssage TO THE Limit IN $m$ AND $\epsilon$. The a priori estimates $(4.25)$ are used now to deduce that there exists $\boldsymbol{u} \in W^{1, \infty}(0, T ; V) \cap W^{2,2}(0, T ; H)$, and a subsequence of $\left\{\boldsymbol{u}_{\epsilon}^{m}\right\}_{m, \epsilon}$ such that for $m \rightarrow \infty$ and $\epsilon \rightarrow 0$, we have:

$$
\begin{gathered}
\boldsymbol{u}_{\epsilon}^{m} \rightarrow \boldsymbol{u} \text { and } \dot{\boldsymbol{u}}_{\epsilon}^{m} \rightarrow \dot{\boldsymbol{u}} \text { weak star in } L^{\infty}(0, T ; V), \\
\ddot{\boldsymbol{u}}_{\epsilon}^{m} \rightarrow \ddot{\boldsymbol{u}} \text { weak in } L^{2}(0, T ; H) .
\end{gathered}
$$

Now, using the convexity argument of the functionals $j$ and the properties of its regularization, the lower semicontinuity arguments and compactness results which are used in [6], one can find that $\boldsymbol{u}$ satisfies

$$
\begin{aligned}
& \int_{0}^{T}\left((\ddot{\boldsymbol{u}}(t), \boldsymbol{w}(t)-\dot{\boldsymbol{u}}(t))_{H}+\widetilde{a}(\boldsymbol{u}(t), \boldsymbol{w}(t)-\dot{\boldsymbol{u}}(t))+c(\dot{\boldsymbol{u}}(t), \boldsymbol{w}(t)-\dot{\boldsymbol{u}}(t))\right) d t \\
& +\int_{0}^{T}(j(\boldsymbol{u}(t), \boldsymbol{w}(t))-j(\boldsymbol{u}(t), \dot{\boldsymbol{u}}(t))) d t \geq \int_{0}^{T}\langle\widetilde{\boldsymbol{f}}(t), \boldsymbol{w}(t)-\dot{\boldsymbol{u}}(t)\rangle_{V^{\prime}, V} d t
\end{aligned}
$$

From the last inequality, we deduce that $\boldsymbol{u}$ is a solution of the variational problem (4.11)-(4.12).

Finally, we define $\varphi$ by (4.13), i.e., $\varphi(t)=B^{-1} \Pi \boldsymbol{u}(t)+B^{-1} q(t), \forall t \in[0, T]$. Having in mind that $B^{-1} \Pi \in \mathcal{L}(V, W), B^{-1} \in \mathcal{L}\left(W^{\prime}, W\right), \boldsymbol{u} \in W^{1, \infty}(0, T ; V)$ and $q \in W^{1,2}\left(0, T ; W^{\prime}\right)$ it is now easy to show that $\varphi \in W^{1,2}(0, T ; W)$ so $(\boldsymbol{u}, \varphi)$ is the unique solution of Problem $P_{V}$ with the regularity (3.20).

ACKNOWLEDGEMENTS.

The authors express their warmest thanks and gratitude to the anonymous referees of this journal for their useful comments and suggestions on the initial version of this paper. 


\section{REFERENCES}

[1] R. C. Batra and J. S. Yang, Saint-Venant's principle in linear piezoelectricity, J. Elasticity 38 (1995), 209-218.

[2] P. Bisenga, F. Lebon and F. Maceri, The unilateral frictional contact of a piezoelectric body with a rigid support, in Contact Mechanics, J. A. C. Martins and Manuel D. P. Monteiro Marques (Eds.), Kluwer, Dordrecht, 2002, 347-354.

[3] G. Duvaut and J. L. Lions, Les inéquations en mécanique et en physique, Dunod, Paris, 1972.

[4] El-H. Essoufi and M. Kabbaj, Existence of solutions of a dynamic Signorini's problem with non local friction for viscoelastic piezoelectric materials, Bull. Math. Soc. Sci. Math. Roumanie (N.S.) 48(96) (2005), 181-195.

[5] T. Ikeda, Fundamentals of Piezoelectricity, Oxford University Press, Oxford, 1990.

[6] I. R. Ionescu and Q-L. Nguyen, Dynamic contact problems with slip-dependent friction in viscoelasticity, Int. J. Math. Comput. Sci, 12 (2002), 71-80.

[7] M. Kabbaj and El-H. Essoufi, Frictional contact problem in dynamic electroelasticity, Glas. Mat. Ser. III 43(63) (2008), 137-158.

[8] F. Maceri and P. Bisegna, The unilateral frictionless contact of a piezoelectric body with a rigid support, Math. Comput. Modelling 28, (1998), 19-28.

[9] R. D. Mindlin, Polarisation gradient in elastic dielectrics, Int. J. Solids Structures 4, (1968), 637-663.

[10] R. D. Mindlin, Continuum and lattice theories of influence of electromechanical coupling on capacitance of thin dielectric films, Int. J. Solids Structures 4 (1969), 11971208.

[11] R. D. Mindlin, Elasticity, piezoelasticity and crystal lattice dynamics, J. Elasticity 4 (1972), 217-280.

[12] M. Sofonea and El-H. Essoufi, A piezoelectric contact problem with slip dependent coefficient of friction, Math. Model. Anal. 9 (2004), 229-242.

[13] M. Sofonea and El-H. Essoufi, Quasistatic frictional contact of a viscoelastic piezoelectric body, Adv. Math. Sci. Appl. 14 (2004), 613-631.

[14] B. Tengiz and G. Tengiz, Some dynamic problems of the theory of electroelasticity, Mem. Differential Equations Math. Phys. 10 (1997), 1-53.

[15] R. A. Toupin, The elastic dielectrics, J. Rational Mech. Anal. 5 (1956), 849-915.

[16] R. A. Toupin, A dynamical theory of elastic dielectrics, Internat. J. Engrg. Sci. 1 (1963), 101-126.

M. Kabbaj

Department of Mathematics

Faculty of Sciences and Techniques

University Moulay Ismail

B. P. 509 - Boutalamine, Errachidia

Morocco

E-mail: kabbaj.mostafa@gmail.com

El-H. Essoufi

Department of Mathematics

Faculty of Sciences and Techniques Settat

University Hassan Premier

Km 3, route Casablanca, B.P. 577, Settat

Morocco

E-mail: essoufi@gmail.com

Received: 29.3.2009.

Revised: 11.5.2009. 